\section{Zur Immunpathogenese des Asthma bronchiale}

Zusammenfassung: Asthma bronchiale ist eine Erkrankung des Respirationstraktes, die mit einer Entzündungsreaktion und Hyperreagibilität des Bronchialsystems gegenüber multiplen exogenen oder endogenen Stimuli assoziiert ist. Neuere Untersuchungen weisen auf die wichtige Rolle von proinflammatorischen Mediatoren und Zytokinen in der Pathogenese des Asthma bronchiale hin, die von Mastzellen, eosinophilen Granulozyten und T-Zellen produziert werden. Durch die verstärkte Expression proinflammatorischer Zytokine kommt es zu einer pulmonalen Entzündungsreaktion, die vor allem durch IL-4, IL-13 und IL-5 vermittelt wird. Aus den neuen Erkenntnissen über die Immunpathogenese des Asthma bronchiale in Tiermodellen hat sich eine Reihe innovativer Therapieansätze ergeben. Diese umfassen die Gabe rekombinanter antiinflammatorischer Zytokine (IL-10), die Wiederherstellung des lokalen Zytokingleichgewichts durch Gabe von TH1-induzierenden Zytokinen (IL-12), die Induktion von TGF-beta produzierenden Zellen und Inhibitoren von IgE sowie von proinflammatorischen Zytokinen (IL-4, IL5) und deren Rezeptoren. Es ist zu erhoffen, dass es durch diese Behandlungsansätze gelingt, selektiver und effektiver in den Krankheitsverlauf eingreifen zu können, als dies bisher durch Gabe von Kortikosteroiden möglich ist. Der klinische Stellenwert der experimentellen Therapieansätze bleibt jedoch abzuwarten; insbesondere sind potenzielle Nebenwirkungen einer systemischen Behandlung mit pluripotenten rekombinanten Zytokinen und/oder Zytokinantagonisten zu untersuchen.

On the Immunopathology of Bronchial Asthma: Allergic asthma is a chronic pulmonary disease associated with bronchoconstriction and inflammation. Recent studies have shown that mediator substances and proinflammatory cytokines produced by mast cells, eosinophils and T-lymphocytes appear to be important for the pathogenesis of asthma. These substances contribute both to the initiation and perpetuation of the disease. In particular, it has been shown that allergic asthma is associated with increased TH2 (IL-4, IL-5, IL-13) cytokine production that causes activation of eosinophils and T-cells and production of chemokines (e.g. eotaxin) by pulmonary fibroblasts. Based on recent advances in our understanding of the immunopathogenesis of asthma in animal models several novel therapeutic approaches have been developed. Such approaches comprise treatment with recombinant anti-inflammatory cytokines, treatment with $\mathrm{TH} 1$-inducing cytokines such as IL-12, induction

Pneumologie 2000; 54: 412-418

(c) Georg Thieme Verlag Stuttgart · New York ISSN 0934-8387

\section{S. Finotto, P. R. Galle, M. F. Neurath}

Labor Immunologie I, Arbeitsgruppe mukosale Immunologie, I. Medizinische Klinik der Johannes Gutenberg Universität Mainz (Direktor: Univ. Prof. Dr. med. P. R. Galle)

of oral tolerance and TGF-beta producing T-cells that can provide bystander suppression for $\mathrm{TH} 2$ cells, inhibitors of $\mathrm{IgE}$, and antagonists of proinflammatory cytokines (e.g. IL-4 and IL-5) and their receptors. These novel treatment modalities will hopefully permit a more selective and effective suppression of pulmonary inflammation and bronchoconstriction in patients with allergic asthma compared to local treatment with corticosteroids. However, the clinical value of these novel therapeutic approaches remains to be determined. In particular, long term efficacy and safety of immunomodulatory therapy has to be studied more in detail.

\section{Ätiologie des Asthma bronchiale}

Asthma bronchiale ist eine Erkrankung des Respirationstraktes, die mit einer Entzündungsreaktion und Hyperreagibilität des Bronchialsystems gegenüber multiplen exogenen oder endogenen Stimuli assoziiert ist [1,2]. Die Häufigkeit der Erkrankung liegt in den industrialisierten Ländern zwischen 5-10\% der Bevölkerung, wobei der Krankheitsbeginn des allergischen Asthmas bei 50\% der Betroffenen vor dem 10 . Lebensjahr und bei einem weiteren Drittel vor dem 40 . Lebensjahr liegt.

Ätiologisch wurden allergische und idiosynkratische bzw. infektassoziierte Asthmaformen beschrieben [2,4,5]. Bei einigen Asthmaformen wird auch eine ätiologische Beziehung zum gastroösophagealen Reflux diskutiert $[2,6]$. Beim allergischen Asthma handelt es sich um immunologisch vermittelte Überempfindlichkeitsreaktionen. Als Hinweis auf eine genetische Prädisposition dieser Patienten sind häufig auch Angehörige von Asthma betroffen und in der Familie treten andere allergische Erkrankungen, wie Rhinitis, Urtikaria und Ekzeme, gehäuft auf. Hinweise auf eine allergisch bedingte Erkrankung geben insbesondere auch positive Hauptprovokationstests mit definierten, die Krankheit auslösenden Allergenen, hohe Serumspiegel für Gesamt-IgE und antigenspezifisches IgE und das Auftreten einer Bronchokonstriktion nach lokaler pulmonaler Antigenexposition. Die Allergene können bereits innerhalb weniger Minuten eine Hyperreagibilität des Bronchialsystems hervorrufen, die vor allem bei hohen Antigendosen über Wochen nachweisbar sein kann $[2,3]$. Neben einer allergischen Sofortreaktion zeigen 30-50\% der Patienten eine zweite Phase der Bronchokonstriktion 6 bis 10 Stunden nach Antigenexposition. Entsprechend der Art der Allergene und der Expositionsmöglichkeit treten oftmals auch saisonale Häufungen asthmatischer Beschwerden auf. 
Pharmaka, die akute Atemwegsbeschwerden induzieren können, sind vor allem Acetylsalicylsäure (ASS) und betaadrenerge Antagonisten (Betablocker). Das typische ASS-sensitive respiratorische Syndrom betrifft meistens Erwachsene und beginnt oft mit einer Rhinitis gefolgt von einer hyperplastischen Rhinosinusitis mit nasaler Polyposis $[1,2,7]$. Im weiteren Verlauf ist dann die Entstehung eines progressiven Asthma bronchiale möglich, bei dem es bereits nach Exposition von kleinen Substanzmengen zu einer nasalen Kongestion und pulmonalen Obstruktion kommt.

Die Prävalenz der Aspirinsensitivität bei Asthmatikern beträgt etwa $10 \%$. Es besteht eine hohe Kreuzreaktivität zwischen Aspirin und zahlreichen anderen nichtsteroiden Antirheumatika (NSAR), wie z.B. Indomethazin, Naproxen, Ibuprofen und Phenylbutazon [2,6]. Eine Desensitivierung ist wie beim allergischen Asthma durch eine tägliche Applikation der Antigene bzw. von ASS möglich. Eine derartige antigenspezifische Immuntherapie führt wahrscheinlich zur Anergie von TZellen und zur Modulation der Zytokinproduktion (s.u.). Neben Pharmaka können auch Chemikalien bei sensibilisierten Personen asthmatische Reaktionen hervorrufen [6].

\section{Immunpathogenese des Asthma bronchiale: Rolle von Mastzellen, eosinophilen Granulozyten und T-Lymphozyten}

Durch zahlreiche Untersuchungen zur Pathogenese des Asthma bronchiale konnte gezeigt werden, dass immunologische Faktoren für die Entstehung asthmatischer Reaktionen von besonders großer Bedeutung sind [2,6]. Während der allergischen asthmatischen Reaktion kommt es zu einer Aktivierung von Mastzellen, Granulozyten, Lymphozyten und Makrophagen, die Mediatorsubstanzen freisetzen, die wiederum direkte Effekte auf lokale Zellen, wie die glatten Muskelzellen, und die Kapillarpermeabilität haben. In der Frühphase der Pathogenese des allergischen Asthmas ist vor allem eine Interaktion von Antigenen mit spezifischen IgE-Antikörpern von Bedeutung, die von Plasmazellen produziert werden und an der Oberfläche von Mastzellen oder basophilen Granulozyten gebunden sind. Nach dieser Interaktion kommt es zu einer Freisetzung von Mediatoren aus Granulozyten und Mastzellen, wie Histamin, Bradykinin, Leukotrienen (C, D und E), Tryptase, PAF und Prostaglandinen, die eine intensive Entzündungsreaktion mit Bronchokonstriktion, Gefäßkongestion und Ödembildung hervorrufen [8,9]. Eine Amplifikation der Entzündung bei Asthma bronchiale kann wahrscheinlich durch die Histaminfreisetzung erfolgen $[2,9]$. Leukotriene können zudem eine erhöhte Mukusproduktion und einen reduzierten Mikrozilientransport bedingen, der zur Erhöhung des Atemwegswiderstandes beiträgt. Chemokine bringen dann Leukozyten, Granulozyten und Blutplättchen zum Ort der Entzündung. Während für die Rekrutierung von eosinophilen Granulozyten Eotaxin wichtig ist, weisen neuere Studien auf die funktionelle Bedeutung von IL-16 für die Rekrutierung von T-Lymphozyten hin [10-12].

Die eosinophilen Granulozyten sind pathogenetisch für die chronische, allergischbedingte Entzündung wichtig, da sie zahlreiche Mediatoren, wie Leukotrien C4 und PAF, produzieren können und daher zur Bronchokonstriktion und Ödembildung beitragen [6]. Durch eine Degranulation von eosinophilen Granulozyten kann es zudem zur Freisetzung von major basic protein (MBP) und eosinophilic cationic protein (ECP) in der Lunge kommen, die die Zilienmotilität reduzieren und zu einer Exfoliation von Zellen in das Bronchiallumen führen [13]. Interessanterweise korreliert die Mediatorfreisetzung aus eosinophilen Granulozyten gut mit dem klinischen Schweregrad der allergischen Erkrankung [14]. Schließlich kommt es durch Mediatorsubstanzen und Entzündungszellen auch zur Schädigung des Lungengewebes und von Nervenendigungen mit Aktivierung axonaler Reflexe, die möglicherweise zur Perpetuierung und Ausbreitung der lokalen pulmonalen Entzündung beitragen. Im Langzeitverlauf kann es zu Änderungen der Lungenstruktur kommen, die die pulmonale Obstruktion verstärken und den Gasaustausch behindern [2]. Zur Verschlechterung der pulmonalen Funktion tragen gegebenenfalls auch zusätzliche Infektionen und eine Exposition zu chemischen Noxen bei.

T-Lymphozyten sind ebenfalls für die Pathogenese des Asthma bronchiale sehr wichtig, da sie zahlreiche Mediatoren bzw. Zytokine produzieren, die die Entzündung verstärken und zur Chronifizierung des Entzündungsgeschehens beitragen können $[2,15,16]$. Bei allergischem Asthma kommt es zu einer T-Helfer 2-(TH2)-Zelldifferenzierung, wobei die TZellen vor allem Zytokine der sog. TH2-Gruppe (vor allem IL4, IL-5, IL-9 und IL-13) produzieren, die direkt lokale Entzündungszellen, wie eosinophile Granulozyten, B-Zellen und TZellen, aktivieren können oder zur Freisetzung von Chemokinen (z. B. Eotaxin) in pulmonalen Fibroblasten führen können $[15,17]$. Ferner sind im Serum der Patienten hohe IL-4-Spiegel zu beobachten [18]. Dagegen findet man bei chronischem Asthma eine reduzierte Produktion von Zytokinen der THelfer 1-Gruppe (IFN-gamma, IL-2) [2,19]. Die hohe Produktion von TH2-Zytokinen ist wahrscheinlich durch eine lokale Aktivierung von TH2-Zellen über spezifische Allergene/Antigene und kostimulatorische Faktoren (z.B. CD40/CD40L, CD28/B7) für T-Zellen bedingt [15,20]. Ferner könnten die bei Asthmapatienten häufig nachzuweisenden TH2-Zytokingenpromotor-Polymorphismen eine genetische Prädisposition für die Entwicklung allergischer Erkrankungen sein. Hierbei ist eine vermehrte transkriptionelle Aktivierung von TH2 (IL-4, IL-9) und TH3 (TGF-beta) Zytokingenpromotoren mit konsekutiver Zytokinproduktion anzunehmen, die zur Induktion und Chronifizierung der allergischen Erkrankung beiträgt bzw. auch über eine vermehrte TGF-beta Produktion die Entstehung einer pulmonalen Fibrose fördert [15,21]. Schließlich weisen neuere Studien darauf hin, dass neben einer pathologischen Aktivierung der TH2-Zytokinproduktion bei Asthma möglicherweise auch Störungen der IL-12-Produktion durch antigenpräsentierende Zellen [19], der IL-12-abhängigen TH1-Zytokinproduktion [19] und der TH1-Zytokinsignaltransduktion vorliegen [22]. Hierbei wurde eine reduzierte IL12- und IFN- $\gamma$-Produktion bei Asthmapatienten beobachtet $[2,19]$. Ferner konnte trotz nur geringer lokaler IFN- $\gamma$ Produktion in der Lunge eine starke Aktivierung des IFN- $\gamma$-abhängigen-Transkriptionsfaktors signal transducer and activator of transcription 1 (STAT-1) in Epithelzellen nachgewiesen werden [22], was auf eine pathologische Zytokinsignaltransduktion hinweist.

Das Auftreten und die funktionelle Bedeutung einer erhöhten TH2 Zytokinproduktion von T-Zellen konnte auch bei In-vivoModellen für bronchiale Hyperreagibilität bzw. asthmatische pulmonale Entzündungsreaktionen bei der Maus gezeigt 
werden [23,24]. Hierbei wird die TH2-Zytokinproduktion durch Präsentation von definierten Antigenen (z.B. Ovalbumin) über antigenpräsentierende Zellen (APC; z.B. Makrophagen, dendritische Zellen, B-Zellen) an T-Lymphozyten induziert, wobei die Interaktion von kostimulatorischen Molekülen wie B7.2 (CD86) auf APC mit CD28 auf T-Zellen eine wichtige Rolle spielt [24]. Zudem konnte durch Untersuchungen an IL-4-transgenen Tieren und IL-4-knockout Mäusen gezeigt werden, dass IL-4 wichtig für die Regulation von TH2Zytokinantworten und die IgE-mRNA-Synthese bei allergischen Erkrankungen ist $[25,26]$. Hierfür sprechen vor allem die hohen IgE-Spiegel und das Auftreten von allergischen Entzündungsreaktionen bei IL-4-transgenen Mäusen, während IL-4-knockout Mäuse eine supprimierte IgE-Produktion aufweisen. Um hohe Mengen an IgE-Protein wie bei Asthma zu erhalten, ist allerdings neben IL-4 ein zweites Signal erforderlich. Dies erfolgt wahrscheinlich durch eine Interaktion von T-Zellen mit APC oder durch kostimulatorische Signale für T-Zellen (möglicherweise über CD40/CD40L und CD28/B7-2). Ferner konnte gezeigt werden, dass auch Mastzellen und basophile Granulozyten selbst die notwendigen Oberflächenstrukturen besitzen, um unabhängig von T-Zellen eine IgE-Synthese durch IL-4 in B-Zellen zu induzieren, so dass auch diese Zellen eine Rolle bei der vermehrten IgESynthese im Rahmen asthmatischer Reaktionen zu spielen scheinen [6]; basierend auf den Erkenntnissen über die Bedeutung von IgE sind anti-IgE-Antikörper mittlerweile auch in klinischen Studien bei Asthma bronchiale getestet worden.

Obwohl viele biologische Funktionen von IL-13 mit denen von IL-4 identisch sind, scheint IL-13 bei allergischen pulmonalen Erkrankungen IL-4-unabhängige pathogene Wirkungen zu haben, wobei dieser Effekt nicht über T-Zellen vermittelt wird $[27,28]$. Ein Aspekt dieser Funktion von IL-13 liegt wahrscheinlich in der Induktion von Chemokinen, wie z.B. Eotaxin, wie in Zellkulturstudien belegt werden konnte [29]. Für die wichtige pathogenetische Funktion von IL-13 sprechen auch Daten aus transgenen Mäusen, die IL-13 selektiv in der Lunge überexprimieren und eine pulmonale Entzündung mit Infiltration von eosinophilen Granulozyten aufweisen [30]. Die vermehrte Produktion des TH2-Zytokins IL-5 bei allergischen asthmatischen Erkrankungen führt zu einer Stimulation von eosinophilen Granulozyten und verhindert wahrscheinlich ihren programmierten Zelltod (Apoptose) im allergischen Gewebe [31]. Daher trägt IL-5 offenbar entscheidend zur Blutund Gewebseosinophilie bei Asthma bei [32]. Ferner kann IL5 eosinophile Granulozyten sensibilisieren, Mediatorsubstanzen freizusetzen [13]. Zu der Rekrutierung und Aktivierung von eosinophilen Granulozyten tragen neben IL-5 jedoch auch noch andere Faktoren, wie Eotaxin und der die Eotaxin bzw. IL-5-Expression kontrollierende Transkriptionsfaktor NF-kappaB p50, bei [33].

\section{Immunmodulatorische Therapieansätze bei Asthma bronchiale: rekombinante Zytokine und Zytokinantagonisten}

Basierend auf Daten aus Tiermodellen haben die oben angeführten Befunde zur Immunpathogenese und Zytokinproduktion auch potenzielle therapeutische Implikationen für Asthmapatienten. Vor allem die Wiederherstellung des Zytokingleichgewichts durch Blockade von TH2-Zytokinen oder Induktion von TH1-Zytokinen ist ein attraktiver Therapieansatz
$[2,6,34]$. Beispielsweise sind Antikörper bzw. lösliche blockierende Zytokinrezeptoren gegen TH2-Zytokine, wie IL-4 und IL-5, verfügbar, die bei Asthma eine wichtige pathogenetische Bedeutung haben $[3,35,36]$. Eine inhibitorische Strategie gegen das letztere Zytokin konnte in einem Tiermodell für allergische Entzündung bereits erfolgreich getestet werden [36]; erste positive Daten liegen mittlerweile auch über den Einsatz von anti-IL-4 Antikörpern bei Patienten mit Asthma bronchiale vor. Analysen in einem Knockout-Modell für den IL-4-abhängigen Transkriptionsfaktor signal transducer and activator of transcription 6 (STAT-6) haben ferner gezeigt, dass eine Suppression der IL-4-Signaltransduktion nicht in der Lage ist, die T-Zellinfiltration in die Lunge zu supprimieren [ 37]. Es kam jedoch zu einer deutlichen Reduktion der eosinophilen Granulozyten in der Lunge, die möglicherweise durch eine reduzierte Aktivierung IL-5 produzierender TH2Zellen bei STAT-6-defizienten Tieren bedingt ist [37].

Zudem wäre eine Induktion von TH1-Zytokinen bei Asthma durch die Gabe von rekombinantem IL-12 denkbar; ein Zytokin das über die Aktivierung des Transkriptionsfaktors signal transducer and activator of transcription 4 (STAT-4) zu einer TH1-T-Zelldifferenzierung führt $[38,39]$. In einem murinen Tiermodell für allergisch bedingte T-Zellaktivierung durch Dermatophagoides pteronyssinus-abstammendes Der $\mathrm{p}$ 1-Allergen war eine Behandlung mit rekombinantem IL-12 hocheffektiv, eine TH2-Zellaktivierung und Eosinophilie zu inhibieren [40]. Eine IL-12-Monotherapie war dagegen in einem ovalbumininduzierten Modell für allergische Entzündung nicht ausreichend, eine antigenspezifische T-Zellaktivierung bei allergisch bedingter bronchialer Hyperreagibilität zu supprimieren, so dass Kombinationstherapien, wie die Applikation von IL-12 plus IL-18, zur Aktivierung von antagonistischen TH1-Zellen bei Asthma vielversprechender erschienen [41]. Die protektive Rolle dieser Zytokinkombination bei bronchialer Hyperreagibilität kann in Zukunft durch die Herstellung und Verfügbarkeit von STAT-4 [38] bzw. IL-18 (Finotto et al., unpublizierte Daten) transgenen Maussystemen genauer untersucht werden. Im Gegensatz $\mathrm{zu}$ einer Behandlung mit TH1-induzierenden Zytokinen war eine Therapie von $\mathrm{TH} 2$-vermittelter allergisch bedingter bronchialer Hyperreagibilität durch Transfer von TH1-Zellen nicht erfolgreich, da diese Zellen selbst zu einer TH1-vermittelten pulmonalen Entzündung führten [42]. In diesem adoptiven Transfersystem waren lediglich TGF-beta produzierende TH3 T-Zellen in der Lage, eine TH2-vermittelte Entzündung effektiv zu supprimieren [42], so dass Mechanismen zur Induktion von TH3-Zellen bzw. von oraler Toleranz ein interessantes Forschungsgebiet beim Asthma bronchiale darstellen.

Ferner wäre eine Behandlung mit dem immunsuppressiv wirkenden Zytokin IL-10 bei allergischem Asthma bronchiale denkbar, zumal es nach antigenspezifischer Immuntherapie bei allergischen Erkrankungen zu einer Induktion von IFN- $\gamma$ und IL-10 kommt $[6,43]$. Auch anti-IgE-Antikörper und 5Lipoxygenaseinhibitoren (BI-L-239) zur Suppression von Leukotrien C4 werden zur Zeit auf ihre tierexperimentelle und klinische Wertigkeit bei allergischen Erkrankungen bzw. Asthmapatienten getestet $[44,45]$. Zudem sind möglicherweise inhibitorische Strategien gegen IL-11 bei virusassoziierten Asthmaformen einsetzbar, da die IL-11-Produktion durch virale Infektionen aktiviert wird und eine erhöhte IL-11Expression im Tiermodell eine Atemwegshyperreaktivität ver- 
ursacht [46]. Schließlich lassen neuere Untersuchungen Störungen in der Apoptose von T-Zellen und eosinophilen Granulozyten bei chronisch eosinophilen Entzündungen bzw. bei Asthma bronchiale vermuten [47-49], so dass auch therapeutische Ansätze zur Induktion einer Apoptose dieser Zellen eventuell klinisch interessant sind.

\section{Neue Therapieansätze bei Asthma bronchiale: Antisense- DNA als Alternative zur Corticosteroidbehandlung}

Bei allergisch bedingtem Asthma bronchiale ist eine Therapie mit Kortikosteroiden sehr effektiv, um die pulmonale Entzündungsreaktion zu supprimieren [2]. Die Ursachen für diesen Effekt sind jedoch erst teilweise bekannt. Kortikosteroide sind pluripotente Moleküle, die nach Bindung an ihren intrazellulären, nukleären Rezeptor hocheffektiv transkriptionelle Vorgänge modulieren können [50]. Hierbei können sie als trans-acting-Faktoren über Bindung an Kortikoid-responsive DNA-Elemente zur Induktion der Gentranskription an zahlreichen Promotoren führen oder auch eine Suppression der transkriptionellen Aktivierung über Inhibierung von Transkriptionsfaktoren vermitteln [50,51]. Bezüglich der Zytokingentranskription können Kortikosteroide eine potente Inhibition der Produktion zahlreicher proinflammatorischer Zytokine (z.B. von IL-1, IFN- $\gamma$, IL-6 und TNF) hervorrufen, die den therapeutischen Effekt von Kortikosteroiden bei Asthma bronchiale erklären könnte. Die Suppression der Zytokingentranskription sowie der Expression von Adhäsionsmolekülen (z.B. ICAM-1, VCAM) beruhen wenigstens teilweise auf einer Kortikosteroidvermittelten Inaktivierung der NF-kappaB- und AP1-abhängigen Gentranskription [50-54]. Hierbei hemmen sie die transkriptionelle Aktivität von DNA-gebundenen AP-1-Familienmitgliedern, bilden mit NF-kappaB p65 einen funktionell inaktiven Komplex oder führen zur transkriptionellen Aktivierung des Promotors des NF-kappaB sequestrierenden IkappaB-Moleküls [50-52].

Kortikosteroide führen jedoch nicht nur zur Modulation der Zytokingenexpression sondern haben auch potente inhibitorische Effekte auf die Expression eines wichtigen Wachstumsund Aktivierungsfaktors für eosinophile Granulozyten und Mastzellen, des sog. Stammzellfaktors (SCF). So konnte gezeigt werden, dass Kortikosteroide die Produktion von SCF in Fibroblasten hemmen, was möglicherweise zur therapeutischen Wirkung dieser Substanzen bei allergischen Erkrankungen, wie dem Asthma bronchiale, beiträgt [53]. In Übereinstimmung mit diesem Konzept konnte kürzlich gezeigt werden, dass eine Antisense-DNA-Strategie gegen SCF zur Suppression der pulmonalen Entzündung und Bronchokonstriktion in einem murinen Asthmamodell führt und dass dieser Effekt mit dem einer Kortikosteroidbehandlung vergleichbar ist [54]. Auch eine andere Arbeitsgruppe konnte kürzlich über den erfolgreichen Einsatz von Antisense-DNA an der Lunge berichten, wobei ein allergeninduziertes Modell für Bronchokonstriktion beim Kaninchen verwendet wurde und das Target der Adenosin-A1-Rezeptor war [55]. Ferner konnte in zwei neueren Studien eine Suppression entzündlicher asthmatischer Reaktionen im Tiermodell durch den Einsatz von Antisense-DNA nachgewiesen werden, wobei C- plus Ghaltige Oligonukleotid-Sequenzen verwendet wurden [56,57]. Von diesen Sequenzen ist bekannt, dass sie eine Induktion der IL-12 Produktion bewirken und über ko-stimulatorische Eigenschaften die Produktion von TH1-Zytokinen induzieren
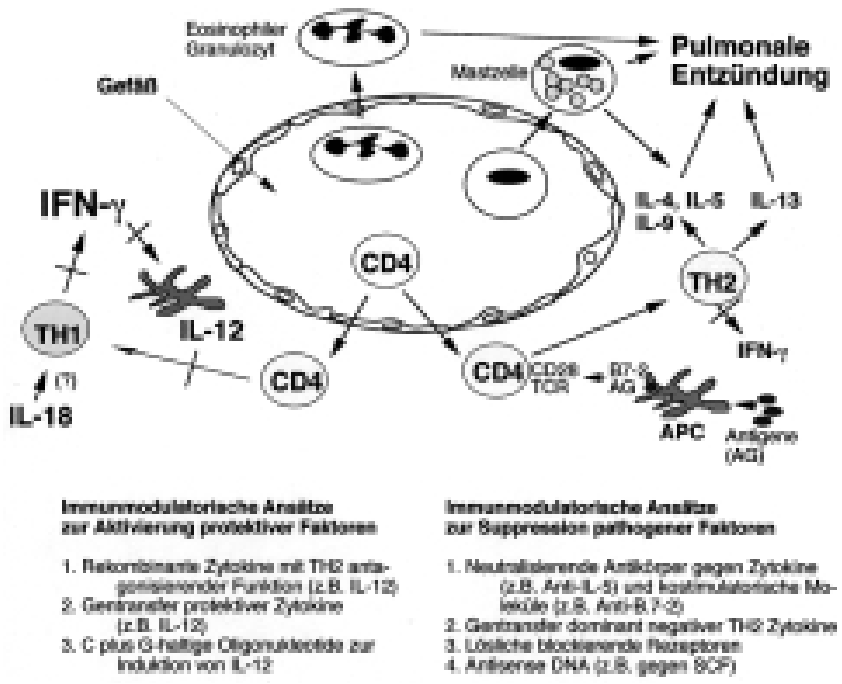

Abb. 1 Hypothetisches Schema der Rolle von Mastzellen, eosinophilen Granulozyten und T-Zellen bei allergischer pulmonaler Entzündung, wie beim Asthma bronchiale. Mastzellen und eosinophile Granulozyten werden im Rahmen der allergischen Reaktion aktiviert und setzen Mediatorsubstanzen frei, die die pulmonale Entzündung verstärken. Mastzellen werden vor allem über die Bindung von spezifischen Antigenen an ihre IgE-Oberflächenantikörper aktiviert. Ferner werden sie über Stammzellfaktor (SCF) aktiviert, der in der Lunge von Fibroblasten und Epithelzellen produziert wird. SCF aktiviert ferner auch eosinophile Granulozyten. Schließlich produzieren T-Zellen in der Lunge bei Asthma nach Antigenexposition TH2Zytokine, die zur pulmonalen Entzündung führen.

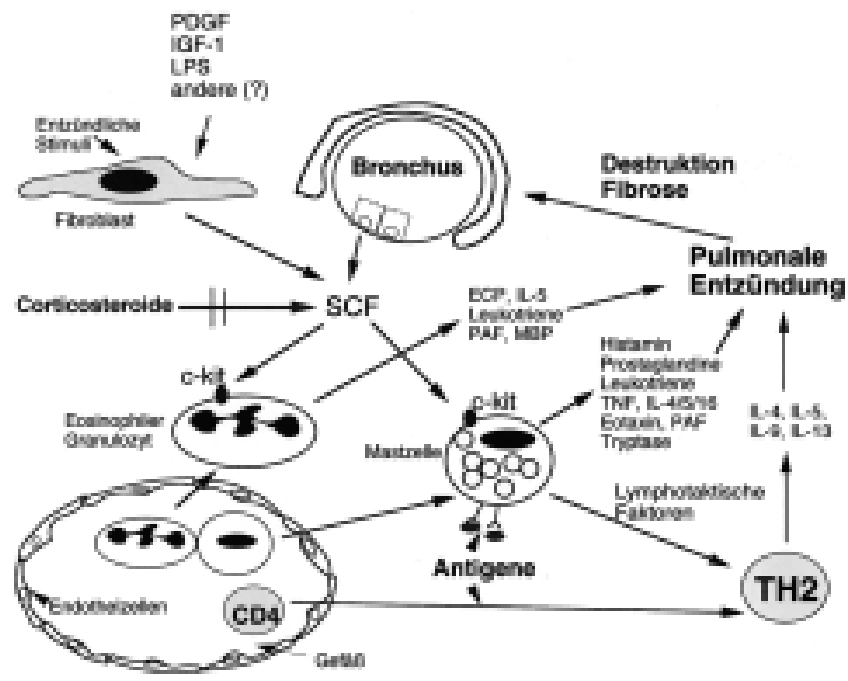

Abb. 2 Rolle von T-Lymphozyten sowie ihrer Mediatoren bei der asthmatischen pulmonalen Entzündung. Die T-Zellen produzieren bei asthmatischen Reaktionen nach Antigenpräsentation über antigenpräsentierende Zellen (APC; über die B7-2/CD28-Interaktion) vor allem die TH2-Zytokine IL-4 und IL-5, jedoch weniger das THI-Zytokin IFN- $\gamma$. Durch diese Verschiebungen des Zytokingleichgewichts wird eine TH2-dominierte pulmonale Entzündung verursacht. Ferner sind mögliche immunmodulatorische Therapien für das Asthma bronchiale aufgeführt. Neben einer antigenspezifischen Therapie sind verschiedene immunmodulatorische Therapieansätze bei asthmatischen pulmonalen Entzündungen denkbar, die rekombinante Zytokine, neutralisierende Antikörper, Antisense-DNA, dominant negative Proteine, blockierende Rezeptorproteine und adenoviralen Gentransfer umfassen $[2,3,6,63-65,68]$. 
[58-60], wodurch der antagonistierende Einfluss dieser Oligonukleotide auf die TH2-vermittelte pulmonale Entzündung verständlich wird. Die Induktion von TH1-Zytokinen durch diese Sequenzen ist hierbei auf molekularer Ebene mit einer Aktivierung von MAP- und JNK-Kinasen und des Transkriptionsfaktors AP-1 assoziiert [60]. Zusammenfassend scheint ein Einsatz von Antisense-DNA-Sequenzen bei Patienten mit Asthma bronchiale daher denkbar, zumal für den möglichen erfolgreichen Einsatz von Antisense-DNA beim Menschen auch erste Daten aus kontrollierten klinischen Studien sprechen $[61,62]$.

Als weitere theoretische Alternative zur Behandlung mit Kortikosteroiden kann ein adenoviraler Gentransfer angesehen werden, bei dem ein Protein oder Antisense-RNA über einen adenoviralen Vektor in der Zelle exprimiert wird. Hierfür spricht beispielsweise die Beobachtung, dass Adenoviren sehr gut in vitro und in vivo in bronchiale Epithelzellen gelangen, was zur Expression von antiinflammatorischen Zytokinen oder anderen Proteinen in der Lunge verwendet werden könnte [63,64]. Bei In-vivo-Studien in einem murinen Asthmamodell konnte ferner gezeigt werden, dass bereits die TH1-dominierte Immunantwort gegen das Adenovirus selbst zur Suppression der pulmonalen TH2-Entzündung beitragen kann [65]. Somit erscheint adenoviraler Gentransfer in der Lunge als ein attraktives neues Therapiekonzept für asthmatische Erkrankungen, das jedoch weiterer Untersuchungen bedarf. Vor allem müssen hierbei die möglichen Risiken einer Immunantwort gegen Adenoviren bedacht werden, zumal in jüngster Zeit über Todesfälle nach adenoviralem Gentransfer beim Menschen berichtet wurde.

Zusammenfassend ergeben sich durch neuere Daten zur Immunpathogenese allergischer Erkrankungen eine Vielzahl neuer Therapieoptionen für die Behandlung von Patienten mit allergisch bedingtem Asthma bronchiale. Es ist zu erhoffen, dass es durch diese Behandlungsansätze gelingt, selektiver und effektiver in den Krankheitsverlauf eingreifen zu können, als dies bisher durch Gabe von Kortikosteroiden möglich ist $[6,66,67]$. Der klinische Stellenwert der neuen experimentellen Therapieansätze bleibt jedoch abzuwarten; insbesondere sind potenzielle Nebenwirkungen einer systemischen Behandlung mit pluripotenten rekombinanten Zytokinen und/ oder Zytokinantagonisten zu untersuchen (Abb. 1, 2).

\section{Danksagung}

Die Autoren möchten sich bei Herrn Prof. Dr. med. Buhl (III. Med. Klinik der Univ. Mainz, Schwerpunkt Pneumologie) für seine Unterstützung und hilfreiche Diskussionen bedanken.

\section{Literatur}

${ }^{1}$ Russi EW. Klinik des Asthma bronchiale. Internist 1999; 40: $825-829$

2 Kaliner MA. Pathogenesis of asthma. In: Clinical Immunology (ed RR Rich). St. Louis: Mosby, 1996: 909-923

${ }^{3}$ Kaliner MA, Lemanske R. Rhinitis and asthma. JAMA 1992; 268: 2807-2811

${ }^{4}$ Howell JBL. Asthma: clinical descriptions and definitions. In: Asthma and rhinitis (eds. Busse WW, ST Holgate). Cambridge: Blackwell Scientific Publishers, 1995: 3-5

${ }^{5}$ Samter M, Beers RF. Intolerance to aspirin. Clinical studies and consideration of its pathogenesis. Ann Intern Med 1968; 68: $975-983$

${ }^{6}$ Simon HU, Blaser K. Immunologische Grundlagen des Asthma bronchiale. Internist 1999; 40: 849-854

${ }^{7}$ Jordana M, Dolovich J, Ohno I, Finotto S, Denburg J. Nasal polyposis: a model for chronic inflammation. In: Asthma and rhinitis (eds. Busse WW, ST Holgate). Cambridge: Blackwell Scientific Publishers, 1995: 156-166

${ }^{8}$ Russi EW, Perruchoud AP, Yerger LD, Stevenson JS, Tabak J, Marchette B, Abraham WM. Late phase bronchial obstruction following nonimmunologic mast cell degranulation. J Appl Physiol 1984; 57: 1182 - 1189

${ }^{9}$ Costa JJ, Galli SJ. Mast cells and basophils. In: Clinical Immunology (ed. RR Rich). 1996: 408 - 430

${ }^{10}$ Mashikian MV, Tarpy RE, Saukkonen JJ, Lim KG, Fine GD, Cruikshank WW. Identification of IL-16 as the lymphocyte chemotactic activity in the bronchoalveolar lavage fluid of histamine-challenged asthmatic patients. J Allergy Clin Immunol 1998; 101: 786-792

${ }^{11}$ Laberge S, Ernst O, Ghaffar O, Cruikshank WW, Komfeld H, Center DM, Hamid Q. Increased expression of interleukin-16 in bronchial mucosa of subjects with atopic asthma. Am J Respir Cell Mol Biol 1997; 17: $193-202$

${ }^{12}$ Hessel EM, Cruikshank WW, vanArk I, DeBie J, vanEsch B, Hofman G, Nijkamp FP, Center DM, Oosterhout AJ. Involvement of IL-16 in the induction of airway hyper-responsiveness and regulation of IgE in a murine model of allergic asthma. J Immunol 1998; 160: 2998-3005

${ }^{13}$ Ackerman SJ. Eosinophils: biologic and clinical aspects in allergy and inflammation. In: Clinical Immunology (ed RR Rich). 1996: $431-448$

${ }^{14}$ Bousquet J, Chanez P, Lacoste LY, Barneon G, Ghavanian N, Enander I, Venge P, Ahlstedt S, Simony-Lafontaine J, Godard P, Michel FB. Eosinophilic inflammation in asthma. N Engl J Med 1990; 323: 1033 - 1039

${ }^{15}$ Kon OM, Kay AB. T cells and chronic asthma. Int Arch Allergy Immunol 1999; 118: 133-135

${ }^{16}$ Gelfand EW. Essential role of T lymphocytes in the development of allergen driven airway hyperresponsiveness. Allergy Asthma Proc 1998; 19: 365-369

17 Teran LM, Mochizuki M, Bartels J, Valencia EL, Nakajima T, Hirai $\mathrm{K}$, Schroeder JM. Th1- and Th2-type cytokines regulate the expression and production of eotaxin and RANTES by human lung fibroblasts. Am J Respir Cell Mol Biol 1999; 20: 1044 - 1549

${ }^{18}$ Miller RL, Eppinger TM, McConnell D, Cunningham-Rundles C, Rothman P. Analysis of cytokine signaling in patients with extrinsic asthma and hyperimmunoglobulin E. J. Allergy Clin Immunol 1998; 102: 503-511

${ }^{19}$ Chou CC, Huang MS, Hsieh KH, Chiang BL. Reduced IL-12 level correlates with decreased IFN-gamma secreting $\mathrm{T}$ cells but not natural killer cell activity in asthmatic children. Ann Allergy Asthma Immunol 1999; 82: 1081 - 1206

${ }^{20}$ Jaffar Z, Roberts K, Pandit A, Linsley P, Djukanovic R, Holgate S. B7 costimulation is required for IL-5 and IL-13 secretion by 
bronchial biopsy tissue of atopic asthmatic subjects in response to allergen stimulation. Am J Respir Cell Mol Biol 1999; 20: $1044-1049$

${ }^{21}$ Rosenwasser LJ. Promoter polymorphism in the candidate genes, EL-4, IL-9, TGF-beta 1, for atopy and asthma. Int Arch Allergy Immunol 1999; 118: $1018-2438$

22 Sampath D, Castro M, Look DC, Holtzman MJ. Constitutive activation of an epithelial signal transducer and activator of transcription (STAT) pathway in asthma. J Clin Invest 1999; 103: $1353-1361$

${ }^{23}$ Henderson WR, Lewis DB, Albert RX, Zhang Y, Lamm WJE, Chiang GKS, Jones F, Eriksen P, Tien Y, Jonas M, Chi EX. The importance of leukotrienes in airway inflanunation in a mouse murine model of asthma. J Exp Med 1996; 184: 1483-1494

${ }^{24}$ Haczku A, Takeda K, Redai I, Hamelmann E, Cieslewicz G, Joetham A, Loader J, Lee JJ, Irvin C, Gelfand EW. Anti-CD86 (B72) treatment abolishes allergic airway hyperresponsiveness in mice. Am J Respir Crit Care Med 1999; 159: 1638-1643

${ }^{25}$ Kopf M, LeGros G, Bachmann M, Lamers MC, Bluethmann H, Koehler G. Disruption of the murine IL-4 gene blocks Th2 cytokine responses. Nature 1993; 362: 245-248

${ }^{26}$ Tepper RI, Levinson DI, Stanger BZ, Campos-Torres J, Abbas AK, Leder P. IL-4 induces allergic-like inflammatory disease and alters $\mathrm{T}$ cell development in transgenic mice. Cell 1990; 62: 457-467

${ }^{27}$ Grünig G, Warnock M, Wakil AE, Venkayya R, Brombacher F, Rennick DM, Sheppard D, Mohrs M, Donaldson DD, Locksley RM, Corry DB. Requirement for IL-13 independently of IL-4 in experimental asthma. Science 1998; 282: 2261 - 2263

${ }^{28}$ Wills-Karp M, Luyimbazi J, Xu X, Schofield B, Neben TY, Karp CL, Donaldson DD. Interleukin-13: central mediator of allergic asthma. Science 1998; 282: 2258-2261

${ }^{29}$ Li L, Xia Y, Nguyen A, Lai YH, Feng L, Mosmann TR, Lo D. Effects of Th2 cytokines on chemokine expression in the lung: IL-13 potently induces eotaxin expression by airway epithelial cells. J Immunol 1999; 162: 2477-2487

${ }^{30}$ Zhu Z, Horner RJ, Wang Z, Chen Q Geba GP, Wang J, Zhang Y, Elias JA. Pulmonary expression of interleukin-13 causes inflammation, mucus hypersecretion, subepithelial fibrosis, physiologic abnormalities, and eotaxin production. J Clin Invest 1999; 103: $779-788$

${ }^{31}$ Simon HU, Yousefi S, Schranz C, Schapowal A, Bachert C, Blaser K. Direct demonstration of delayed esoinophil apoptosis as a mechanism causing tissue eosinophilia. J Immunol 1997; 158: $3902-3908$

32 Robinson DS, North J, Zeibecoglou K, Ying S, Meng Q, Rankin S, Hamid Q Tavemier J, Kay AB. Eosinophil development and bone marrow and tissue eosinophils in atopic asthma. Int Arch Allergy Immunol 1999; 118: 98 - 100

${ }^{33}$ Yang L, Cohn L, Zhang DH, Homer R, Ray A, Ray P. Essential role of nuclear factor kappaB in the induction of eosinophilia in allergic airway inflammation. J Exp Med 1998; 188: 1739-1750

${ }^{34}$ Pauwels RA, Brusselle GG, Tournoy KG, Lambrecht BN, Kips JC. Cytokines and their receptors as therapeutic targets in asthma. Clin Exp Allergy 1998; 28: 1 - 5

${ }^{35}$ Garrone P, Djossou O, Galizzi JP, Banchereau J. A recombinant extrace Hular domain of the human interleukin-4 receptor inhibits the biological effects of interleukin-4 on $\mathrm{T}$ and $\mathrm{B}$ lymphocytes. Eur J Immunol 1991; 21: 1365 - 1369

36 Danzig M, Cuss F. Inhibition of interleukin-5 with a monoclonal antibody attenuates allergic inflammation. Allergy 1997; 52: $787-794$

${ }^{37}$ Miyata S, Matsuyama T, Kodama T, Nishioka Y, Kuribayashi K, Takeda K, Akira S, Sugita M. STAT-6 deficiency in a mouse model of allergen-induced airways inflammation abolishes eosinophi- lia but induces infiltration of CD8+ T-cells. Clin Exp Allergy 1999; 29: 114-123

${ }^{38}$ Wirtz S, Finotto S, Kanzler S, Lohse AW, Blessing M, Lehr HA, Galle PR, Neurath MF. Chronic intestinal inflammation in STAT-4 transgenic mice: characterization of disease and adoptive transfer by TNF- plus IFN-gamma-producing CD4+ T-cells that respond to bacterial antigens. J Immunol 1999; 162: 1884-1888

${ }^{39}$ Magnan A, vanPee D, Bongrand P, Vervloet D. Alveolar macrophage interleukin (EL)-10 and IL-12 production in atopic asthma. Allergy 1998; 53: $1092-1095$

${ }^{40}$ Lee YL, Fu CL, Ye YL, Chiang BL. Administration of interleukin-12 prevents mite Der pl allergen-1gE antibody production and airway eosinophil infiltration in an animal model of airway inflammation. Scand J Immunol 1999; 49: 229-236

${ }^{41}$ Hofstra CL, vanArk L, Hofman G, Kool M, Nijkamp FP, Oosterhout AJ. Prevention of Th2-like cell responses by coadministration of EL-12 and EL-18 is associated with inhibition of antigen-induced airway hyperresponsiveness, eosinophilia, and serum IgE levels. J Immunol 1998; 161: $5054-5060$

${ }^{42}$ Hansen G, Berry G, DeKruyff RH, Umetsu DT. Allergen-specific Thl cells fail to counterbalance Th2 cell-induced airway hyperreactivity but cause severe airway inflammation. J Clin Invest 1999; 103: $175-183$

${ }^{43}$ Jutel M, Pichler WJ, Skrbic D, Urwyler A, Dahinden C, Müller UR. Bee venom immunotherapy results in decrease of IL-4 and EL-5 and increase of IFN-gamma secretion in specific allergen stimulated T cell cultures. J Immunol 1995; 154: 4187-4194

${ }^{44}$ Heusser C, Jardieu P. Therapeutic potential of anti-IgE antibodies. Curr Opin Immunol 1997; 9: 805-814

${ }^{45}$ Wegner CD, Gundel RH, Abraham WM. The role of 5-lipoxygenase products in preclinical models of asthma. J Allergy Clin Immunol 1993; 91: $917-925$

${ }^{46}$ Einarsson O, Geba GP, Zhu Z, Landry M, Elias JA. Interleukin-11: stimulation in vivo and in vitro by respiratory viruses and induction of airways hyperresponsiveness. J Clin Invest 1996; 97: $915-924$

${ }^{47}$ Simon HU, Yousefi S, Dommann-Scherrer C, Zimmermann DR, Bauer S, Barandum J, Blaser K. Expansion of cytoldne-producing CD4-CD8-T cells associated with abnormal Fas expression and hypereosinophilia. J Exp Med 1996; 183: 1071 - 1082

${ }^{48}$ Hebestreit H, Dibbert B, Balatti I, Braun D, Schapowal A, Blaser K, Simon HU. Disruption of Fas receptor signaling by nitric oxide in eosinophils. J Exp Med 1998; 187: 415-425

${ }^{49}$ Spinozzi F, Agea E, Fizzotti M, Bassotti G, Russano A, Droetto S, Bistoni O, Grignani F, Bertotto A. Role of T-helper 2 cytokines in down-modulation of fas mRNA and receptor on the surface of activated CD4+ T-cells: molecular basis for the persistence of the allergic immune response. FASEB J 1998; 12: 1747- 1753

50 Beato M, Herrlich P, Schütz G. Steroid hormone receptors: many actors in search of a plot. Cell 1995; 83: $851-857$

${ }^{51}$ Auphan N, DiDonato JA, Rosette C, Helmberg A, Karin M. Immunosuppression by glucocorticoids: inhibition of NF-kB activity through induction of IkB synthesis. Science 1995; 270: $286-290$

${ }^{52}$ Scheinman RI, Cogswell PC, Lofquist AK, Baldwin AS. Role of transcriptional activation of IkBa in mediation of immunosuppression by glucocorticoids. Science 1995; 270: 283-286

${ }^{53}$ Finotto S, Mekori YA, Metcalfe DD. Glucocorticoids decrease tissue mast cell number by reducing the production of the c-Idt ligand, stem cell factor, by resident cells. In vitro and in vivo evidence in murine systems. J Clin Invest 1997; 99: 1721 - 1729

${ }^{54}$ Finotto S, Bürke M, Lindau K, Schmitt E, Galle PR, Neurath MF. Local administration of Antisense phosphorothioate oligonucleotides to stern cell factor abrogates airway inflammation and TH2 cytokine production in a murine model of asthma. Submitted for publication. 1999 
${ }^{55}$ Nyce LW, Metzger LW. DNA Antisense therapy for asthma in an animal model. Nature 1997; 385: 721 - 725

${ }^{56}$ Broide D, Schwarze J, Tighe H, Gifford T, Nguyen MD, Malek S, VanUden J, Martin-Orozco E, Gelfand EW, Raz E. Immunostimulatory DNA sequences inhibit IL-5, eosinophilic inflammation, and airway hyperresponsiveness in mice. J Immunol 1999; 161: $7054-7062$

${ }^{57}$ Sur S, Wild JS, Choudhury BJ, Sur N, Alam R, Klinman DM. Long terin prevention of allergic lung inflanunation in a mouse model of asthma by CpG oligonucleotides. J Immunol 1999; 162: $6284-6293$

${ }^{58}$ Lipförd GB, Heeg K, Wagner H. Bacterial DNA as immune cell activator. Trends Microbiol 1998; 5: 496-500

${ }^{59}$ Bendigs S, Salzer U, Lipford GB, Wagner H, Heeg K. CpG oligodeoxynucleotides co-stimulate primary $\mathrm{T}$ cells in the absence of antigen-presenting cells. Eur J Immunol 1999; 36: $1209-1218$

${ }^{60}$ Hacker H, Mischak H, Miethke T, Liptay S, Schmid R, Sparwasser T, Heeg K, Lipford GB, Wagner H. CpG-DNA-specific activation of antigen-presenting cells requires stress kinase activity and is preceded by non-specific endocytosis and endosomal maturation. EMBO J 1998; 17: 6230 - 6240

${ }^{61}$ Yacyshyn BR, Bowen-Yacyshyn MB, Jewell L, Tami JA, Bennett CF, Kisner DL, Shanahan WR. A placebo-controlled trial of ICAM-1 Antisense oligonucleotide in the treatment of Crohn's disease. Gastroenterology 1998; 121: 1133-1142

${ }^{62}$ Hanania EG, Kavanagh J, Hortobagyi G, Giles RE, Champlin R, Deisseroth $\mathrm{AB}$. Recent advances in the application of gene therapy to human disease. Am J Med 1995; 59: 537-552

${ }^{63}$ Raczka E, Kukowska-Latallo JF, Rymaszewski M, Chen C, Baker JR. The effect of synthetic surfactant Exosurf on gene transfer in mouse lung in vivo. Gene Ther 1998; 8: 1333-1339

${ }^{64}$ Lee LH, Zabner J, Welsh MJ. Delivery of an adenovirus vector in a calcium phosphate coprecipitate enhances the therapeutic index of gene transfer to airway epithelia. Hum Gene Ther 1999; 9: $603-613$

65 Stampfli MR, Ritz SA, Neigh GA, Sime PJ, Lei XF, Xing Z, Croitoru $\mathrm{K}$, Jordana $\mathrm{M}$. Adenoviral infection inhibits allergic airways inflammation in mice. Clin Exp Allergy 1998; 28: 1581 - 1590

${ }^{66}$ Barnes PJ, Adcock IM. Transcription factors in asthma. Eur Respir J 1998; 12: $221-234$

${ }^{67}$ Atsuta J, Plitt J, Bochner BS, Schleimer RP. Inhibition of VCAM-1 expression in human bronchial epithelial cells by glucocorticoids. Am J Respir Cell Mol Biol 1999; 20: 1044-1549

${ }^{68}$ Rocken M, Racke M, Shevach EM. IL-4-induced immune deviation as antigen-specific therapy for inflanunatory autoimmune disease. Immunol Today 1996; 17: 225-231
Priv.-Doz. Dr. Dr. rer. nat. S. Finotto

I. Medizinische Klinik der Universität Mainz Langenbeckstraße 1

55131 Mainz

E-mail: finotto@mail.uni-mainz.de 